\title{
EFFETS DES ALCOOLS GRAS SUR LA FÉCONDATION ET LES GAMĖTES DE LA TRUITE ARC-EN-CIEL
}

\author{
R. BILLARD \\ Laboratoire de Physiologie des Poissons \\ 78350 JOUY EN JOSAS
}

\section{INTRODUCTION}

L'épandage d'alcools gras sur divers plans d'eau a été envisagé afin de limiter l'évaporation et prévenir ainsi la formation de brouillard. De telles substances peuvent présenter, pour la faune et la flore aquatiques, un certain nombre de dangers qu'il est nécessaire d'identifier avant de procéder à leur utilisation. Dans la présente étude, les effets d'alcools gras comme l'hexadécanol et l'octodécanol ont été recherchés sur la survie des gamètes de truite arc-en-ciel (Salmo gairdneri) et sur la fécondation.

\section{1 - MATERIEL ET METHODES}

La méthode consiste à utiliser des lots de 200 ovules environ, fécondés par différentes concentrations de sperme dans le dilueur d'insémination artificielle (dilueur $532=0532$ ) auquel on a ajouté les produits à tester.

\section{A) Exposition des ovules}

Dans ce dilueur dont la composition minérale, la pression osmotique et le $\mathrm{pH}$ sont contrôlés (BILLARD, 1977), le micropyle de l'ovule demeure ouvert et les ovules peuvent rester plusieurs heures dans ce dilueur tout en conservant leur fécondité. Dans l'eau douce, au contraire le micropyle de l'ovule se ferme en moins d'une minute. Le D 532 convient donc pour exposer les ovules des salmonidés à l'action d'un polluant avant la fécondation. 
B) Exposition du sperme après dilution dans le milieu minèral du liquide séminal (MMLS)

Dans ce milieu, riche en potassium (BILLARD et JALABERT, 1974), les spermatozoides demeurent immobiles. Lorsque I'on diminue de moitie la concentration en potassium, en ajoutant le D 532, les spermatozoides entrent en motilité et peuvent féconder des ovules. Dans ce milieu, le sperme immobile peut survivre plusieurs heures. Le MMLS convient donc pour exposer les spermatozoides à l'action d'un polluant en phase liquide.

\section{C) Protocole expérimental}

L'hexadécanol et l'octodécanol sont mis en suspension dans le D 532 ou le MMLS à l'aide d'un broyeur "ultra-turrax. Les ovules sont exposés pendant 20 à $40 \mathrm{mn}$ à différentes concentrations d'alcool gras $(1,10,100$ ou $1000 \mathrm{mg} / \mathrm{l})$ avant d'être inséminés. Le sperme, étudié à deux dilutions dans le MMLS $\left(10^{-3}\right.$ et $10^{-4}$ ) est exposé aux mêmes concentrations en alcool gras que les ovules pendant 20 ou $40 \mathrm{mn}$.

Afin de tester les effets des alcools gras sur les spermatozoĩdes mis en mouvement, le sperme est dilué dans le D 532 contenant les produits à tester et le mélange est ainsi laissé pendant une minute avant l'addition des ovules. Le sperme, dans ces conditions, entre en mouvement et sa survie est mesurée après une minute de motilité ; la fécondance du sperme peut être modifiée par des polluants et on dispose alors d'un test biologique original.

L'effet des alcools gras sur les gamètes de la truite est aussi étudié au moment de la fécondation, lorsque les ovules et le sperme sont mis simultanément en présence dans le D 532 .

Pour s'affranchir des variations individuelles de la qualité des gamètes, nous avons groupé et mélangé les ovules et le sperme d'une dizaine de géniteurs. L'insémination est pratiquée sous couvert à température constante $\left(10^{\circ} \mathrm{C}\right)$. Après la fécondation, les œufs sont incubés en conditions thermorégulées à $10^{\circ} \mathrm{C}$ pendant 10 jours puis fixés et observés pour déterminer le pourcentage de développement embryonnaire à ce stade, ce qui correspond à une estimation du pourcentage de fécondation.

\section{2 - RESULTATS}

(fig. tabl.)

A) Effet de l'acool gras sur les gamètes au moment de la fécondation :

L'octodécanol n'induit pas de diminution significative du pourcentage de fécondation, même à la concentration de $1 \mathrm{~g} / \mathrm{l}$, quelle que soit ia dilution du sperme utilisee. Par contre. l'hexadécanol, à la concentration de $1 \mathrm{~g} / \mathrm{l}$, diminue significativement $(P<0.05)$ le pourcentage de fécondation lorsque les ovules sont inséminés avec du sperme dilué à $10^{-4}$; tableau (A). (fig. tabl.)

B) Effet des alcools gras sur la survie du sperme motile dans le D 532 :

Après une minute d'exposition dans le $D$ 532, la survie du sperme n'est affectée ni par Thexadécanol ni par l'octodécanol; tableau (B). 
C) Effet des alcools gras sur la survie du sperme non motile dans le MMLS; tableau (C).

A la plus forte dilution utilisée, $10^{-4}$, les deux alcools gras ont un effet négatif sur la survie du sperme dès la plus faible concentration testée $(1 \mathrm{mg} / \mathrm{l})(\mathrm{P}<$ 0.01). A la dilution de $10^{-3}$, la chute du pouvoir fécondant du sperme n'apparait qu'à la plus forte concentration testée ( $\mathrm{g} / \mathrm{l})$.

D) Effet des alcools gras sur la survie des ovules dans le D 532.

L'aptitude à la fécondation des ovules n'est pas modifièe après une exposition à $1 \mathrm{~g} / \mathrm{l}$ d'hexadécanol, mais diminue significativement $(P<0.01)$ après la mise en présence pendant $40 \mathrm{mn}$ à $100 \mathrm{mg} / \mathrm{l}$ d'octodécanol.

Tableau : Effet des alcools gras sur la fertilité des gamètes de la truite arc-enciel (Salmo gairdneri). Analyse statistique Test : $X^{2}$ (d.I 4)

(NS) : non significatif.

(S) : significatif.

\begin{tabular}{|c|c|c|c|c|c|}
\hline No & $\begin{array}{l}\text { Traitement } \\
\text { des gamètes }\end{array}$ & $\begin{array}{c}\text { Durée } \\
\text { d'exposition } \\
(m n)\end{array}$ & $\begin{array}{c}\text { Dilution } \\
\text { du } \\
\text { sperme }\end{array}$ & $\begin{array}{l}\text { Hexa- } \\
\text { décanol }\end{array}$ & $\begin{array}{l}\text { Octo- } \\
\text { décanol }\end{array}$ \\
\hline$\hat{\imath}$ & Insémination & 4 & $10^{-3}$ & $\begin{array}{l}2.422 \\
\text { (NS) }\end{array}$ & $\begin{array}{l}0.206 \\
\text { (NS) }\end{array}$ \\
\hline A & DIA 532 & 1 & $10^{-4}$ & $\begin{array}{l}45.800 \\
(S)\end{array}$ & $\begin{array}{l}2.778 \\
\text { (NS) }\end{array}$ \\
\hline$B$ & $\begin{array}{l}\text { Spermatozoides } \\
\text { motiles } \\
\text { DIA } 532\end{array}$ & 1 & $10^{-3}$ & $\begin{array}{c}59.064 \\
(S)\end{array}$ & $\begin{array}{c}15.094 \\
(S)\end{array}$ \\
\hline \multirow{4}{*}{$C$} & \multirow{4}{*}{$\begin{array}{l}\text { Spermatozoides } \\
\text { non motiles } \\
\text { (MMLS) }\end{array}$} & \multirow{2}{*}{20} & $10^{-3}$ & $\begin{array}{c}310.273 \\
\text { (S) }\end{array}$ & $\begin{array}{c}64.923 \\
\text { (S) }\end{array}$ \\
\hline & & & $10^{-4}$ & $\begin{array}{l}507.235 \\
\text { (S) }\end{array}$ & $\begin{array}{c}434.539 \\
(S)\end{array}$ \\
\hline & & \multirow{2}{*}{40} & $10^{-3}$ & $\begin{array}{c}261.417 \\
(S)\end{array}$ & $\begin{array}{c}1.886 \\
\text { (NS) } \\
\end{array}$ \\
\hline & & & $10^{-4}$ & $\begin{array}{c}156.557 \\
(\mathrm{~S})\end{array}$ & $\begin{array}{c}60.668 \\
\text { (S) }\end{array}$ \\
\hline \multirow{2}{*}{$D$} & \multirow{2}{*}{$\begin{array}{l}\text { Ovisies dans } \\
\text { le DiA } 532\end{array}$} & 20 & $10^{-3}$ & $\begin{array}{c}17.42 \\
(\mathrm{~S}) \\
\end{array}$ & $\begin{array}{l}1.756 \\
\text { (NS) }\end{array}$ \\
\hline & & 40 & $10^{-3}$ & $\begin{array}{l}13.763 \\
(\mathrm{~S})\end{array}$ & $\begin{array}{l}38.118 \\
(S)\end{array}$ \\
\hline
\end{tabular}




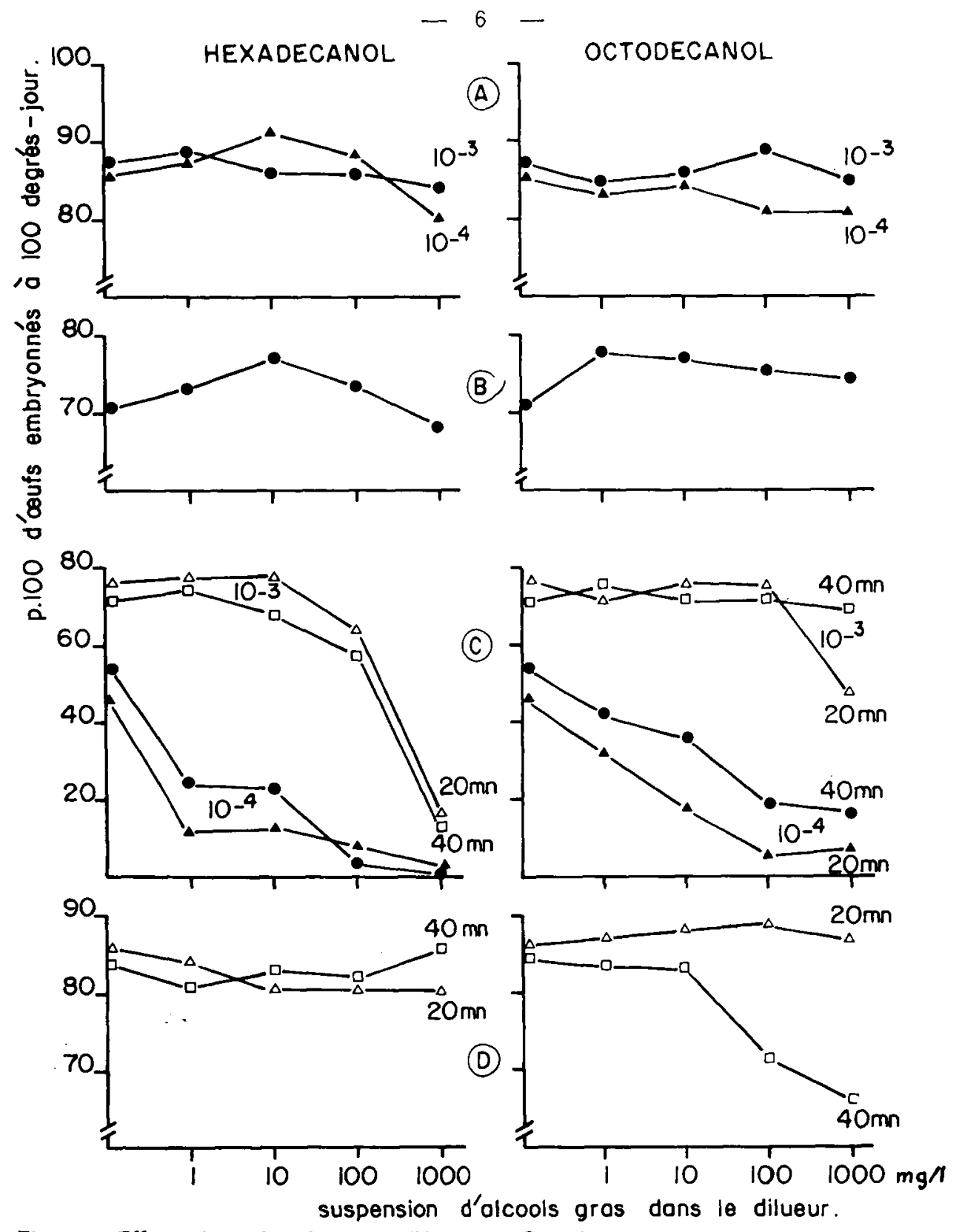

Fig. : Effets des alcools gras (Hexa et Octodécanol) sur les gamètes de la truite arc en ciel.

A : Mise en présence simultanée du sperme et des ovules dans le dilueur d'insémination artificielle (D 532) contenant différentes concentrations d'hexadécanol ou d'octodécanol. Deux concentrations de sperme : dilution au $1 / 1000\left(10^{-3}\right)$ et $1 / 10000\left(10^{-4}\right)$.

B : Exposition du sperme motile pendant $1 \mathrm{mn}$ avant l'insémination dans le D 532.

C : Exposition du sperme non motile pendant 20 ou $40 \mathrm{mn}$ à deux concentrations $10^{-3}$ et $10^{-4}$ (dilueur : MMLS)

D : Exposition des ovules pendant 20 et $40 \mathrm{mn}$ dans le dilueur D 532 Insémination avec du sperme dilué au $1 / 1000\left(10^{-3}\right)$. 


\section{DISCUSSION}

La méthode consistant à exposer des gamètes de poissons à un produit ou à un traitement thermique a déjà étè utilisée (BILLARD et GILLET 1975, ROSENTHAL et ALDERDICE 1976). Dans le cadre de la présente expérience, des résultats montrent que le sperme de truite arc-en-ciel est sensible à l'action d'alcool gras comme l'octodécanol ou l'hexadécanol après des durées d'exposition de 20 et $40 \mathrm{mn}$. Les effets sont amplifies par la dilution, puisque la diminution du pouvoir fécondant intervient à la dose de $1 \mathrm{mg} / \mathrm{l}$ pour une dilution de 1/10000 et de $1 \mathrm{~g} / /$ pour une dilution de 1/1000. Par contre, des durées d'exposition aux alcools gras plus brèves ( $1 \mathrm{mn}$ ), sur des spermatozoides motiles, n'affectenr pas la fécondance du sperme. MANN et SCHMID (1961) ont observé de leur côté que l'exposition du sperme de truite â divers détergents conduisait à une diminution de motilité et du pouvoir fécondant des spermatozoìdes à partir de concentrations de 5 à $10 \mathrm{ppm}$. La fécondabilité des ovules de truite arc-en-ciel n'est diminuée que par des doses très importantes d'octodécanol $(0,1 \mathrm{~g} / \mathrm{l})$, tandis que l'hexadécanol n'a aucun effet, mẽme à la dose de $1 \mathrm{~g} / \mathrm{l}$. II semble donc que l'hexadécanol soit moins toxique que l'octodécanol sur les ovules, alors que le phènomène inverse s'observe pour le sperme après 20 et $40 \mathrm{mn}$ d'exposition à faible dilution.

\section{REMERCIEMENTS}

Ce travail a été financé par la Météorologie Nationale (Contrat no 65 1851).

\section{RESUME}

Des ovules de truite arc-en-ciel (Salmo gairdneri) ont été exposés séparément (mesure des effets sur les gamètes) ou simultanément (mesure des effets sur la fécondation) à des doses croissantes d'hexadécanol et d'octodécanol. L'hexadécanol après une durée d'exposition de 20 et $40 \mathrm{mn}$ provoque sur le sperme immobile une diminution significative $(P<0.01)$ du pouvoir fécondant qui est p'us élevèe à la plus forte dilution ( $1 \mathrm{mg} / \mathrm{l}$ pour une dilution de $1 / 10000$ et $1 \mathrm{~g} / \mathrm{l}$ pour une dilution de 1/1000). L'octodécanol ne modifie le pouvoir fécondant que dans le cas des fortes dilutions Une durée d'exposition d'une minute sur du sperme motile ne modifie pas le pouvoir fécondant. La fécondabilité des ovules est diminuée significativement $(P<0.01)$ après exposition pendant $40 \mathrm{mn}$ à $100 \mathrm{mg} / \mathrm{d}$ d'octodécanol mais n'est pas modifiée par une exposition de même durée à $1 \mathrm{~g} / \mathrm{l}$ d'hexadécanol. Les gamètes mis en présence des alcools gras au moment de la fécondation ne sont affectés qu'aux fortes doses $(1 \mathrm{~g} / \mathrm{l})$ d'hexadécanol.

\section{SUMMARY}

Rainbow trout eggs and milt were exposed separately (effects on gametes) or simultaneously (effects on fertilization) to increased doses of hexadecanol and octodecanol. Hexadecanol induced a significant drop $(P<0.01)$ of the fertilizing ability of sperm after 20 and $40 \mathrm{mn}$ exposure which is more important in the case of higher dilutions $(1 \mathrm{mg} / \mathrm{l}$ at $1 / 10000$ dilution and $1 \mathrm{~g} / /$ at $1 / 1000)$. Octodecanol changed the sperm fertilizing ability only in the case of high dilution. One minute exposure of octodecanol and hexadecanol on motile spermatozoa did not modify fertilizing ability. Eggs fertility was significantly reduced $(P<0.01$ ) following 40 $\mathrm{mn}$ exposure at $100 \mathrm{mg}$ !! of octodecano! but not at $1 \mathrm{~g} / 1$ of hexadecanol. When fatty alcohols were added at the time of insemination, changes occured in the percentage of fertilization only for the highest dose of hexadecanol (1 $\mathrm{g} / \mathrm{l})$. 


\section{BIBLIOGRAPHIE}

BILLARD R., 1977. Utilisation d'un système tris-glycocolle pour tamponner le dilueur d'insémination pour truite. Bull. Franc. Pisc., 264, 103-112.

BILLARD R., JALABERT B., 1974 L'insemination artificielle de la truite (Salmo gairdneri Richardson). Ann. Biol. anim. Bioch. Biophys., 14, 601-610.

BILLARD R., GILLET G., 1975. Effets de la température sur la fécondation et la survie des gamètes chez la truite arc-en-ciel. Bull. Franc. Pisc., 259, 53-65.

MANN H., and SCHMID O.J., 1961. Der Einfluss von Detergentien auf Sperma, Befruchtung und Entwiklung bei der Forelle. Int. Rev. Ges Hydrobiol., 46, $282-$ 300.

ROSENTHAL H., ALDERDICE D.F., 1976. Sublethal effects of environmental stressors, natural and pollutional, on marine fish eggs and larvae. J. Fish. Res. Bd Can., 33, 2 047-2 065. 